\title{
Identification of a five-gene signature for predicting the progression and prognosis of stage I endometrial carcinoma
}

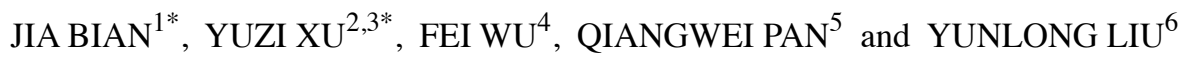 \\ ${ }^{1}$ Department of Gynecology and Obstetrics, Yinzhou Hospital Affiliated to Medical School of Ningbo University, Ningbo, \\ Zhejiang 315040; ${ }^{2}$ Department of Oral Implantology and Prosthodontics, The Affiliated Stomatology Hospital, Zhejiang University \\ School of Medicine; ${ }^{3}$ Key Laboratory of Oral Biomedical Research of Zhejiang Province, Zhejiang University School of \\ Stomatology, Hangzhou, Zhejiang 310006; ${ }^{4}$ School of Medicine, Anhui University of Science and Technology, \\ Huainan, Anhui 232001; 5 Department of Gynecology and Obstetrics, Wenzhou People's Hospital, \\ Wenzhou, Zhejiang 325000; ${ }^{6}$ Department of Medical Oncology, Sir Run Run Shaw Hospital, \\ Zhejiang University School of Medicine, Hangzhou, Zhejiang 310016, P.R. China
}

Received December 19, 2019; Accepted May 28, 2020

DOI: 10.3892/ol.2020.11798

\begin{abstract}
Uterine corpus endometrial carcinoma (UCEC) is often diagnosed at an early clinical stage based on abnormal vaginal bleeding. However, the prognosis of UCEC is poor. The present study was conducted to identify novel tumor grade-related genes with the potential to predict the prognosis and progression of UCEC. A total of three gene expression microarray datasets were downloaded from the Gene Expression Omnibus database, and one RNA-sequencing dataset with corresponding clinical information of patients with UCEC was obtained from The Cancer Genome Atlas database. In summary, 1,447 differentially expressed genes (DEGs) were identified between endometrial cancerous tissues and normal endometrial tissues. Weighted gene co-expression network analysis was performed to assess the associations between DEGs and clinical traits. In total, five genes were found to be highly associated with the tumorigenesis and prognosis of UCEC. Among them, BUB1 mitotic checkpoint serine/threonine kinase B, cyclin B1, cell-division cycle protein 20 and non-SMC condensing I complex subunit $G$ were involved in cell cycle regulation pathways, and DLG-associated protein 5 was involved in the Notch receptor 3 signaling pathway based on functional enrichment analyses. Of the five genes,
\end{abstract}

Correspondence to: Dr Jia Bian, Department of Gynecology and Obstetrics, Yinzhou Hospital Affiliated to Medical School of Ningbo University, 251 Baizhang East Road, Ningbo, Zhejiang 315040, P.R. China

E-mail: 923416944@qq.com

${ }^{*}$ Contributed equally

Key words: uterine corpus endometrial carcinoma, weighted gene co-expression network analysis, prognosis, tumor grade, survival analysis four were highly expressed in endometrial cancerous tissues compared with normal endometrial tissues at the protein level. In addition, the higher expression of these genes predicted a higher tumor grade and worse overall survival. In conclusion, the present study revealed a 5-gene signature that can be used to predict the progression of UCEC.

\section{Introduction}

Uterine corpus endometrial carcinoma (UCEC) is one of the most common cancer types that affects women worldwide (1). According to the 2009 International Federation of Gynecology and Obstetrics classification, stage I or stage II UCEC is categorized as early stage UCEC $(2,3)$. In total, $75 \%$ of UCEC cases are diagnosed at stage I of the disease. At this early stage, patients clinically present with abnormal vaginal bleeding (4). Due to early diagnosis, the 5-year survival rate of patients with UCEC is $29 \%$ (5). However, in a subset of patients diagnosed with early stage UCEC, the disease manifests in a poorly differentiated form and is classified as grade III UCEC (6). High-grade stage I UCEC progresses rapidly and presents with a high metastatic rate within a short time (6). Higher-grade classification takes into account the number of tumor cells undergoing differentiation, which allows the more malignant biological behavior to be observed (7). High-grade UCEC frequently presents with lymph-vascular space invasion and deep myometrial infiltration (8). Therefore, there is an urgent need to investigate the underlying mechanisms that drive the tumorigenesis and progression of stage I UCEC. The therapeutic strategy employed in the treatment of UCEC depends on the tumor grade. For stage I UCEC, surgical intervention is preferred, and total hysterectomy and removal of oviduct and ovaries are standard treatments (6). For high-grade UCEC, adjuvant treatments, such as radiotherapy and chemotherapy, are preferred, as they achieve improved outcomes $(9,10)$. Due to its high recurrence rate, the 5-year overall survival (OS) rate of grade 3 UCEC ranges between 20 and $40 \%$ (11). Therefore, novel and specific targeted therapies with fewer negative 
side effects are urgently needed to improve the outcomes for patients with high-grade stage I UCEC.

It has previously been reported that the PI3K/AKT/mTOR signaling axis is frequently dysregulated in UCEC (12). However, a trial testing the clinical efficacy of mTOR inhibitors against UCEC revealed a response rate of $<10 \%$ (13). Other therapeutic strategies have also been evaluated, including use of inhibitors of PI3K (14), vascular endothelial growth factor/fibroblast growth factor receptor $(15,16)$, poly (ADP-ribose) polymerase (17) and programmed cell death protein 1/programmed death-ligand 1 (18), with outcomes showing the limited efficacy of these agents.

Advances in the application of high-throughput microarray technology in research, coupled with the availability of public databases, has made bioinformatics analysis a powerful means for identifying critical biomarkers in cancer (19-21). However, to the best of our knowledge, very few studies $(22,23)$ have investigated the interconnection among genes. By contrast, numerous studies have focused on the identification of differentially expressed genes (DEGs) between cancer and normal tissues (24-26). Weighted gene co-expression network analysis (WGCNA) allows the identification of associations between genes and the clinical features of cancer based on scale-free networks (27). WGCNA has previously been used to identify genes associated with clinical features of cancer, such as stage, tumor grade and metastasis (28-30).

In the present study, WGCNA was performed on microarray data obtained from the Gene Expression Omnibus (GEO) database and UCEC RNA-sequencing (RNA-seq) data from The Cancer Genome Atlas (TCGA). The publicly available data were screened to identify DEGs for determination of their association with tumor grade. The present study may help to identify novel tumor grade-associated genes with the potential to predict the prognosis and progression of UCEC, which may provide insight for an improved understanding of UCEC pathogenesis.

\section{Materials and methods}

Data source. A workflow diagram for the present study is shown in Fig. 1. The following datasets were downloaded from the publicly available endometrial carcinoma (EC) GEO database (http://www.ncbi.nlm.nij.gov/geo/): GSE17025 (31), GSE56026 (32) and GSE115810 (33). Datasets GSE17025 and GSE56026 were based on the Affymetrix Human Genome U133 Plus 2.0 Array, while dataset GSE115810 was based on the Affymetrix Human Genome U133A Array (both Affymetrix; Thermo Fisher Scientific, Inc.). The GSE17025 dataset, which consists of 91 stage I EC samples and 12 normal endometrium samples, was used to identify the DEGs that are associated with tumor grade. This GSE17025 dataset consisted of 30 grade I samples, 36 grade II samples and 25 grade III samples. An UCEC RNA-seq dataset and its corresponding clinical information was also downloaded from TCGA database (https://www.cancer.gov/tcga) using the following inclusion criteria: i) Histological diagnosed UCEC; ii) no other malignancy except UCEC; and iii) available detailed clinical and follow-up information. This dataset contains 581 samples, of which 35 are normal endometrial tissue samples and 546 are UCEC samples. This TCGA dataset was used to investigate the expression levels of the DEGs and to analyze OS. The GSE115810 and GSE56026 datasets were used as test datasets for the validation of the association of DEG expression levels with various tumor grades.

Data preprocessing. A quality control analysis of the raw microarray data was performed using the 'Affy' package (34) for $\mathrm{R}$ software ( $\mathrm{R}$ version 3.6) using the following functions: The relative logarithmic expression (RLE) and the normalized unscaled standard errors (NUSE) (35). Data were considered to be of sufficiently high quality if the box plot center was at $\sim 0$ for the RLE function and $\sim 1$ for the NUSE function. Data that failed to meet the quality threshold were excluded from downstream analysis. Next, raw microarray data were subjected to background correction, normalization and logarithmic conversion using the Robust Multi-Array Average function in the 'Affy' package for $\mathrm{R}$ software ( $\mathrm{R}$ version 3.6) (36).

Identification of DEGs. Subsequently, the gene expression profile data from the GSE17025 dataset were analyzed using the 'limma' package (37) for $\mathrm{R}$ software ( $\mathrm{R}$ version 3.6 ). This was performed to identify DEGs in EC compared with normal endometrial samples. Only candidate genes within the cut-off limit of $\mid \log _{2}$ fold-change (FC) $\mid \geq 1$ and an adjusted P-value of $<0.05$ were selected for further analysis.

Construction of co-expression network. To construct a co-expression network, a matrix was assembled with the gene symbols as column names and expression profiles as the row names. Another matrix was assembled using the corresponding clinical information with the similar approach. Next, normal endometrium samples and UCEC samples with missing data or genes with zero variance, were all removed using the goodSamplesGenes function of the 'WGCNA' package in $\mathrm{R}$ software ( $\mathrm{R}$ version 3.6). Following this step, the 'WGCNA' package was employed to construct a co-expression network of the DEGs, as described previously (27). Briefly, Pearson's correlation matrices were conducted for all pair-wise genes. Next, power functions were applied to establish a weighted adjacency matrix. The following function was used: $\mathrm{a}_{\mathrm{mn}}=\left|\mathrm{c}_{\mathrm{mn}}\right| \beta$, where $\mathrm{a}_{\mathrm{mn}}$ indicates adjacency between gene $\mathrm{m}$ and gene $\mathrm{n}$, and $\mathrm{c}_{\mathrm{mn}}$ indicates Pearson's correlation between gene $m$ and gene $n$. The best power ( $B$ value) was applied as a soft-thresholding parameter that permitted emphasis on strong DEG correlations while penalizing weak correlations (38). Next, adjacencies were transformed into topological overlap matrices to minimize interference from noise and spurious associations (39). Any genes exhibiting identical expression profiles were grouped together into gene modules using hierarchical clustering. Minimum module size was set at 30 genes as previously described (40). Finally, the dissimilarity of module eigengenes (MEs) was calculated and a cutoff threshold of 0.25 was applied to merge highly identical modules together. Principal component analysis was employed to identify MEs as the major component for each module.

Elucidation of tumor grade-associated modules. Next, the MEs, together with the corresponding clinical features, were used for module-trait relationship analysis in $\mathrm{R}$ software ( $\mathrm{R}$ version 3.6) (38). To this end, Pearson's correlation 


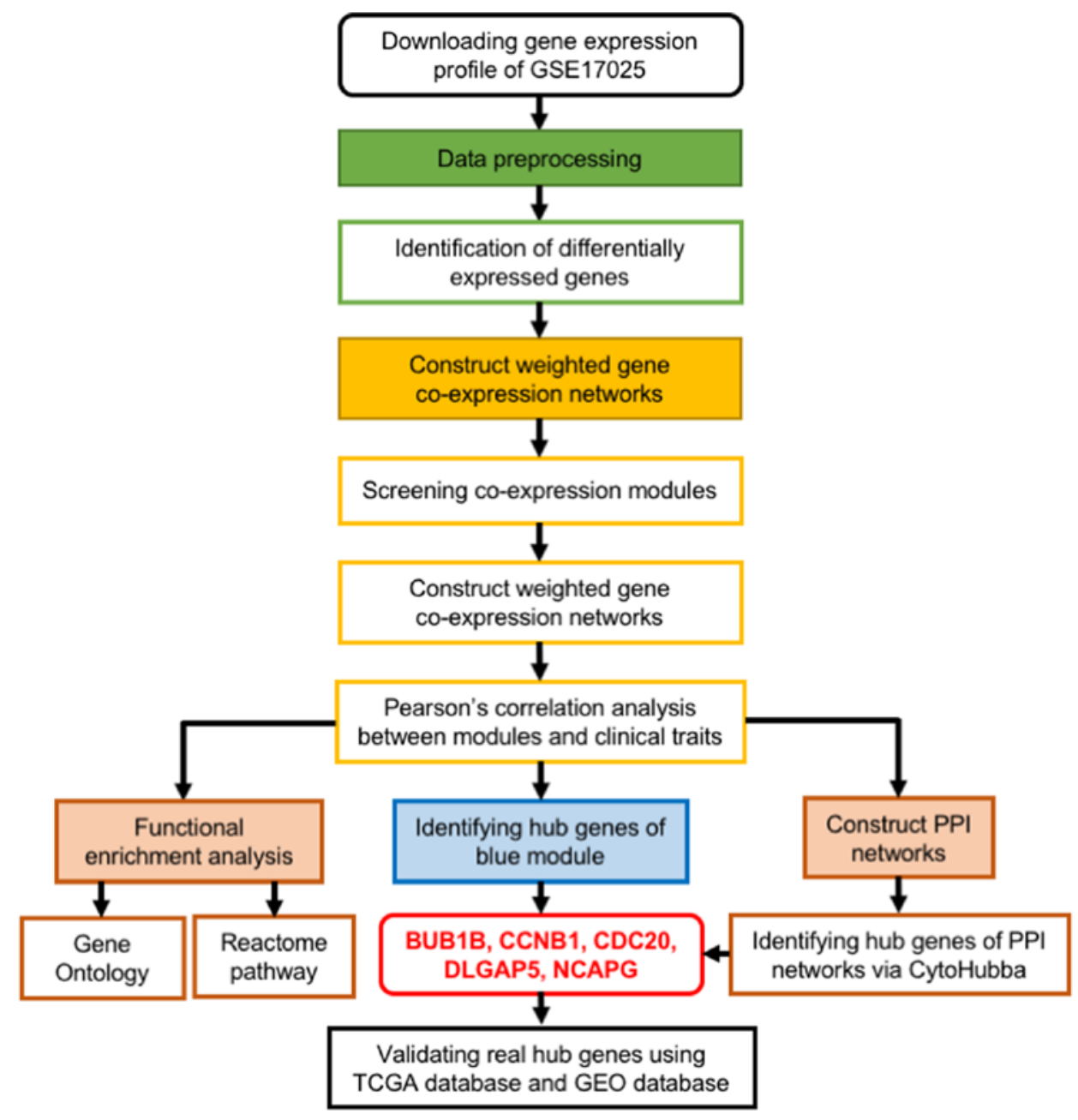

Figure 1. Flow chart outlining the data preparation, processing, analysis and validation in the present study. PPI, protein-protein interaction; BUB1B, BUB1 mitotic checkpoint serine/threonine kinase B; CCNB1, cyclin B1; CDC20, cell-division cycle protein 20; DLGAP5, DLG-associated protein 5; NCAPG, non-SMC condensing I complex subunit; TCGA, The Cancer Genome Atlas; GEO, Gene Expression Omnibus.

coefficients and P-values were calculated and plotted into a heatmap to clearly visualize any tumor grade-related modules. Genes that were associated with clinical features of the samples were also calculated. These genes were used to calculate module significance, which is given as the mean of the individual gene significance values for the genes present in a given module. Finally, the 'highest module', which is the module with the strongest correlation with clinical features of UCEC, was identified.

Functional enrichment analyses. Next, functional enrichment analyses were performed to identify the biological processes associated with the DEGs. This was achieved using Gene Ontology (GO) analysis of all genes in the highest module with the 'clusterProfiler' package (41) in R software (R version 3.6). For this analysis, the statistical cutoff threshold was set at $\mathrm{P}<0.05$. Genes in the highest threshold were also subjected to Reactome pathway analysis $(42,43)$. The pathway enrichment threshold was set at a false discovery rate $<0.05$.

Identification of hub genes. Modules were further analyzed to identify module hub genes. Hub genes are defined as those genes that exhibit the highest correlation with certain clinical features (38). Hub genes were selected from the highest module based on module membership (correlation between the MEs and the gene expression profiles) and gene significance (correlation between genes and certain UCEC clinical features). Module memberships and gene significance were both derived using the absolute values from Pearson's correlation analysis in $\mathrm{R}$ software ( $\mathrm{R}$ version 3.6). The following criteria were used to select hub genes: cor.geneModuleMembership $>0.8$ and cor.geneTraitSignificance $>0.3$ (38). Next, STRING (version 11; https://string-db.org/) (44) was used to construct protein-protein interaction (PPI) networks for genes of interest selected from the highest module. To visualize the results of this analysis, Cytoscape (version 3.7.0; http://www.cytoscape. org/) (45) was used, applying a minimum required interaction score of 0.700. Hub genes were identified and highlighted using CytoHubba (46), a Cytospace plug-in. To prioritize potential hub genes, the following methods were employed in CytoHubba: Maximal clique centrality (MCC), maximum neighborhood component (MNC), degree, edge percolated component (EPC) and bottleneck (BN). The top 20 genes, returning the highest connectivity score using each method were considered to be hub genes. To further narrow the list of potential hub genes, the lists generated using the respective 
methods were combined and the overlapping candidates were selected as the real hub genes.

Validation and survival analysis based on TCGA database. UCEC RNA-seq data for 581 samples were downloaded from TCGA database. This dataset was used to analyze the expression levels of real hub genes in normal compared with tumor tissues. First, the best expression cut-off threshold was set for each gene. This was achieved by taking the gene expression value with the highest survival difference between the two groups as the lowest Renyi test P-value. Next, the 541 UCEC samples for which corresponding clinical information was available were split into high and low expression groups. Kaplan-Meier (KM) survival analysis was performed to determine the associations between real hub genes and OS rate. The Human Protein Atlas (http://www.proteinatlas.org) (47) was employed to map the tissue protein expression pattern for the real hub genes.

Statistical analysis. The R software (R version 3.6) was used to identify DEGs, screen out key modules and perform functional enrichment analyses $(27,37,41)$. The associations between clinical features and MEs were assessed by Pearson's correlation analysis in R software ( $\mathrm{R}$ version 3.6) (38). Subsequently, the following statistical analyses were performed using GraphPad Prism 7 (version 7.0a; GraphPad Software, Inc.). KM was used to analyze OS, and the differences between the study groups were compared using the Renyi test (48). To evaluate the associations between expression levels of real hub genes and UCEC tumor grade, an unpaired two-tailed Student's t-test or one-way ANOVA followed by Tukey's post-hoc test were performed for data comparisons. All values are presented as the mean \pm standard deviation. $\mathrm{P}<0.05$ was considered to indicate a statistically significant difference.

\section{Results}

Identification of DEGs. Following preprocessing and normalization of gene expression data, gene expression matrices were generated for the 103 samples in the GSE17025 dataset. To match clinical features with sample name, a dendrogram was generated using one of the cluster methods named 'complete' from the 'WGCNA' package in R software. Clinical features for the 91 tumor samples, including age, ethnicity, histology, disease stage and tumor grade characteristics, were derived from this dataset (Fig. 2A). In the reported cases, the mean age of patients with EC was $60.80 \pm 10.71$ years, the oldest patient was 91 years old and the youngest was 37 years old. In terms of ethnicity, 68 patients were Caucasian, 18 were African American, 2 were American Indian, 1 was Asian and 1 was Hispanic (data from 1 patient was missing). The histological pattern of 79 cases was typical of the endometrioid EC type, while that of the other 12 cases was typical of the papillary serous EC type. Excluding 1 case with stage I EC that could not be further subdivided into stages, the other 90 cases consisted of 30 stage IA cases, 37 stage IB cases and 23 stage IC cases. Next, the samples were categorized into EC tissues or normal tissues based on indicated pathology. After applying a cut-off threshold of $\mid \log _{2} \mathrm{FCl} \geq 1$ and an adjusted $\mathrm{P}<0.05,1,447$ DEGs, including 439 upregulated DEGs and 1,008 downregulated DEGs, were identified (Fig. 2B and C).
Construction of a weighted co-expression network and identification of key modules. The expression levels of 1,447 DEGs from 91 UCEC samples and the corresponding clinical information were assembled into matrices for co-expression analysis. To ensure a scale-free network, a power of $\beta=3$ (scale free $\mathrm{R}^{2}=0.9$ ) was applied as soft-thresholding (Fig. 3A and B). From this analysis, nine key modules were identified for downstream analysis (Fig. 3C). From this analysis, the blue module (highest module) demonstrated the highest correlation with tumor grade $\left(\mathrm{r}=0.54 ; \mathrm{P}=4 \times 10^{-8} ;\right.$ Fig. $\left.3 \mathrm{D}\right)$. The highest module contained 428 tumor grade-related genes $\left(\mathrm{r}=0.73 ; \mathrm{P}=2 \times 10^{-72}\right.$; Fig. 3E).

The 'clusterProfiler' package in R software was then used to conduct GO enrichment analysis to identify the biological processes associated with the DEGs. It was identified that the DEGs were involved in 'chromosome segregation' $\left(\mathrm{P}=3.830 \times 10^{-27}\right)$, 'nuclear division' $\left(\mathrm{P}=1.993 \times 10^{-25}\right)$, 'organelle fission' $\left(\mathrm{P}=1.694 \times 10^{-24}\right)$ and 'mitotic nuclear division' $\left(\mathrm{P}=2.428 \times 20^{-24}\right.$; Fig. 4A). Pathway analysis revealed that 216 out of 428 genes of the highest module were associated with the 'cell cycle, mitotic' pathways $\left(\mathrm{P}=1.11 \times 10^{-16}\right)$, the 'cell cycle' $\left(\mathrm{P}=1.11 \times 10^{-16}\right)$, 'cell cycle checkpoint' $\left(\mathrm{P}=2.12 \times 10^{-11}\right)$ and 'mitotic spindle checkpoint' $\left(\mathrm{P}=4.693 \times 10^{-10}\right)$. Out of the 728 enriched pathways, the top 25 pathways are presented in Table I. The pathway enrichment threshold was set at a false discovery rate $<0.05$.

Identification of hub genes. A total of 60 hub genes exhibiting high connectivity in the blue module were identified with cor. geneModuleMembership $>0.8$ and cor.geneTraitSignificance $>0.3$. A PPI network of all genes in the blue module was generated using Cytoscape to visualize interactions among the genes. This revealed 178 nodes and 2,435 edges. Of the 178 nodes, 134 genes (red nodes) were upregulated, whereas 44 genes (purple nodes) were downregulated. For each node, the intensity of color was proportional to the level of gene expression in the tumor compared with non-tumor tissues (Fig. 4B). To identify hub genes, the following CytoHubba plug-in methods were employed: MCC, MNC, degree, EPC and BN. From these analyses, the top 20 hits produced by each method were considered as hub genes. The hub gene lists produced by each method were then combined and the overlapping candidates were selected as the real hub genes for further analysis. These real hub genes were BUB1 mitotic checkpoint serine/ threonine kinase B (BUB1B), cyclin B1 (CCNB1), cell-division cycle protein 20 (CDC20), DLG-associated protein 5 (DLGAP5) and non-SMC condensing I complex subunit G (NCAPG) (Fig. 5A).

Validation of hub gene expression levels. Subsequently, the five real hub genes were subjected to validation analysis. To do so, the expression levels of the five genes were first analyzed in the GSE17025 dataset. The results demonstrated that all five genes were highly expressed in UCEC tissues compared with normal tissues. In addition, patients who had more advanced tumor grades exhibited significantly higher expression levels of the five genes compared with patients with lower grade tumors (Fig. 5B). In the testing datasets GSE115810 and GSE56026, BUB1B, DLGAP5 and NCAPG expression demonstrated significant association with tumor grade in the GSE56026 
A Sample dendrogram and trait heatmap

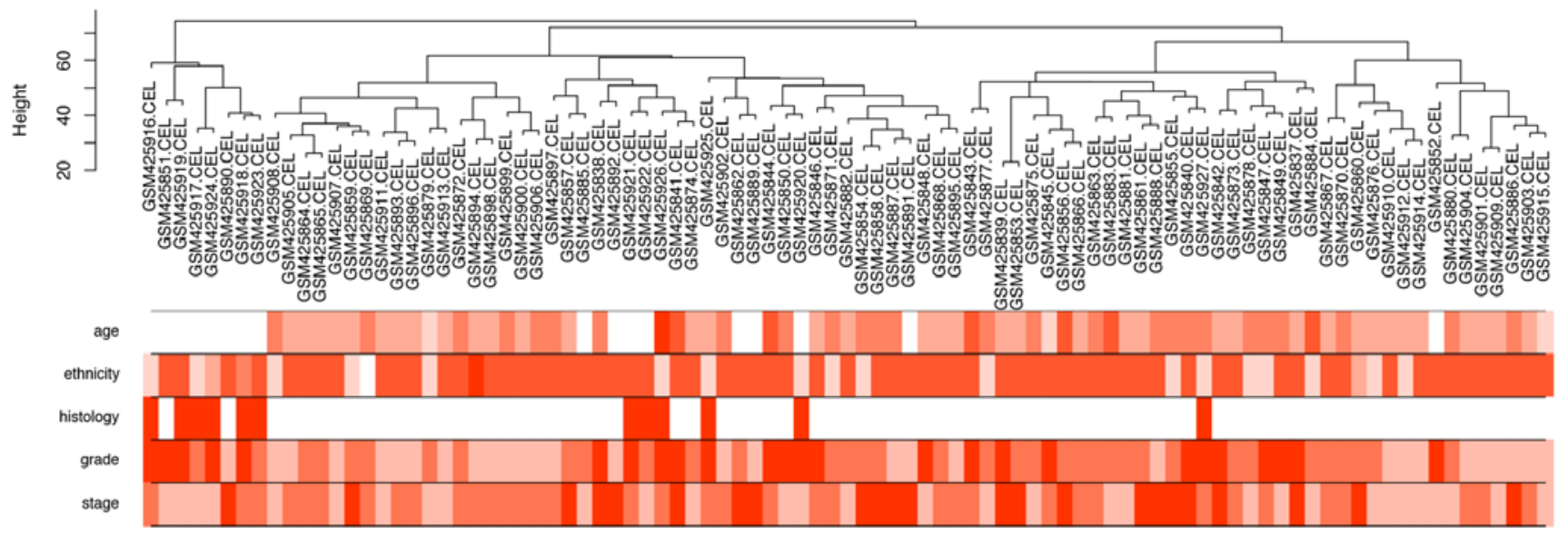

$\mathrm{B}$

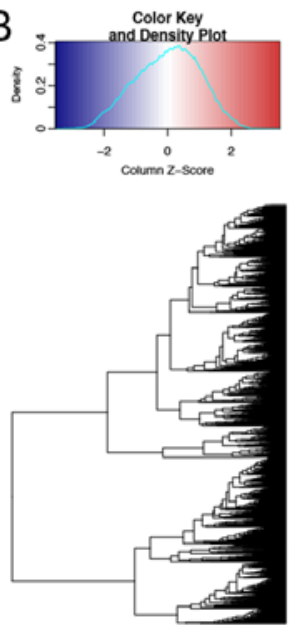

Gene expression's heatmap of GSE17025

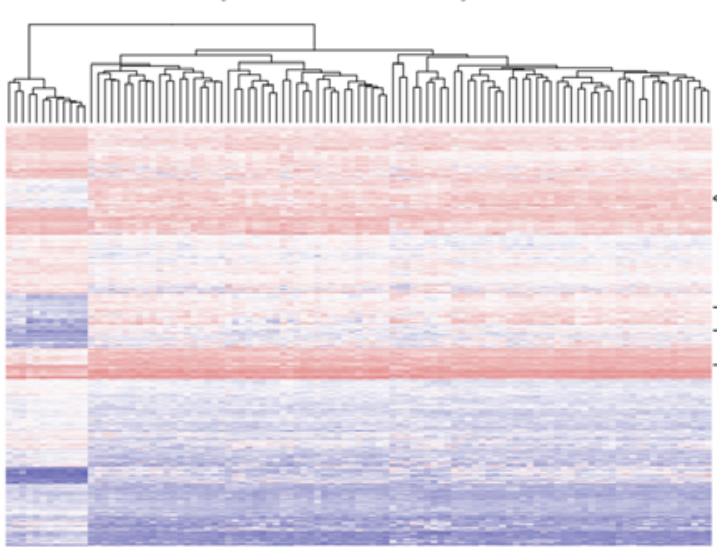

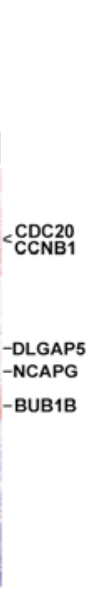

C DEGs in endometrial cancer

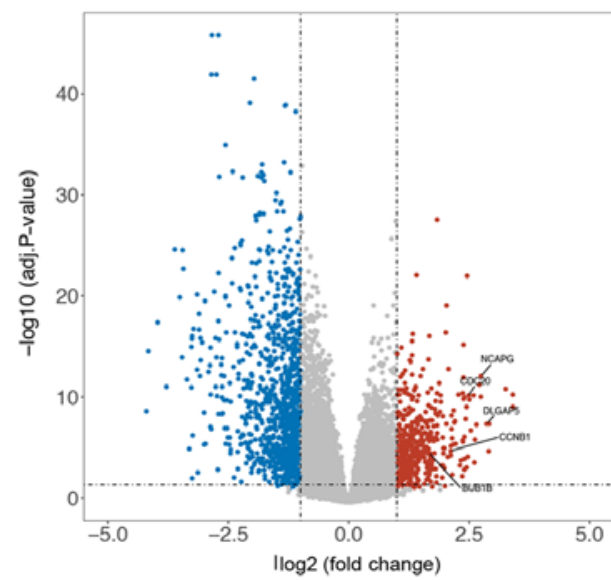

Figure 2. Identification of DEGs in GSE17025. (A) Clinical traits of 91 tumor samples from the GSE17025 dataset. White represents missing age, missing ethnicity and endometrioid histology data. Red for histology represents papillary serous endometrial carcinoma. The color intensity is proportional to the age, tumor grade and pathological stage, and the alphabetical order of ethnicity (African American, American Indian, Asian, Caucasian and Hispanic). (B) A heatmap plot showing gene expression levels of 103 samples from the GSE17025 dataset. Red indicates a high gene expression level and blue indicates a low gene expression level. The color intensity is proportional to gene expression level. (C) A volcano plot of DEGs in GSE17025. The upregulated DEGs selected based on the cut-off criteria ( $\mid \log 2$ fold-changel $\geq 1$ and adj. $\mathrm{P}<0.05$ ) are shown in red, and the downregulated DEGs are shown in blue. DEG, differentially expressed gene; adj., adjusted; BUB1B, BUB1 mitotic checkpoint serine/threonine kinase B; CCNB1, cyclin B1; CDC20, cell-division cycle protein 20; DLGAP5, DLG-associated protein 5; NCAPG, non-SMC condensing I complex subunit.

dataset, while in the GSE115810 dataset, CDC20 exhibited a significant association with tumor grade (Fig. 5C and D). To further evaluate the clinical significance of the five genes, the associations between gene expression levels and clinical traits, including clinical stage, histological diagnosis and age, were investigated using the GSE17025 dataset. All five genes were expressed as at significantly higher level in stages Ia, Ib and Ic compared with those in normal tissues; however, no significant differences were observed in the expression of these genes among stage Ia, Ib and Ic EC samples (Fig. 5E). In terms of tumor histology, there were no significant differences in gene expression levels between endometrioid EC and papillary serous EC (Fig. 5F). In addition, the gene expression levels were not correlated with the age of the patients (Fig. 5G).

Reactome pathway analysis for the hub genes. The 5 hub genes identified in the aforementioned step were also subjected to Reactome pathway analysis, which revealed that BUB1B, CCNB1, CDC20 and NCAPG were involved in cell cycle processes and particularly in the 'mitotic prometaphase' pathway. DLGAP5 was associated with the NOTCH3 intracellular domain, a component of the Notch pathway involved in gene transcription processes (Table II) (49).

Survival analysis based on TCGA database. To validate the aforementioned results, TCGA RNA-seq data for 546 UCEC samples and 35 normal endometrial tissue samples were used for further analyses. The gene expression levels of the five real hub genes were compared between normal endometrial tissue and UCEC tissue. Consistent with results from GEO analysis, it was identified that all real hub genes were expressed at a significantly higher level in UCEC samples compared with those in normal endometrial tissue samples (Fig. 6A-E). Subsequently, the best expression cut-off point for each real hub gene was set and the 541 UCEC samples for which clinical information was available were separated into two groups that yielded maximal difference with regard to survival at the lowest Renyi test P-value. BUB1B ( $\mathrm{P}=0.0053)$, CCNB1 
A

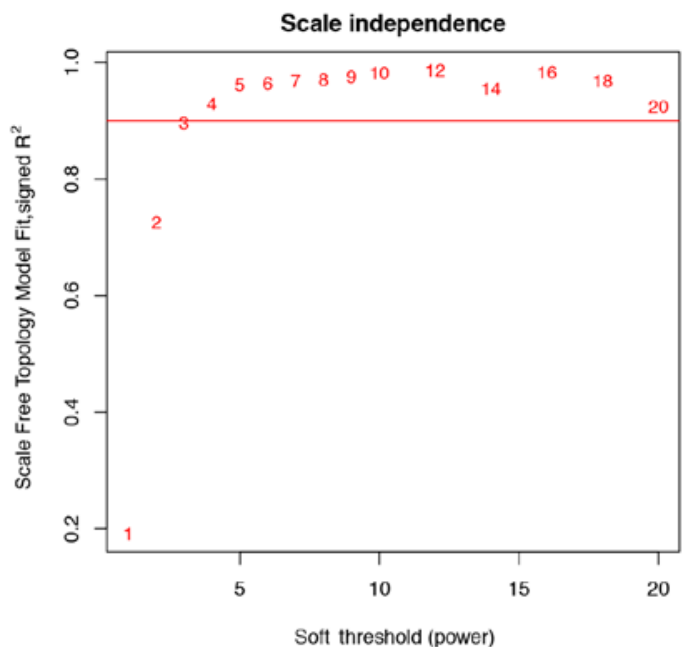

B

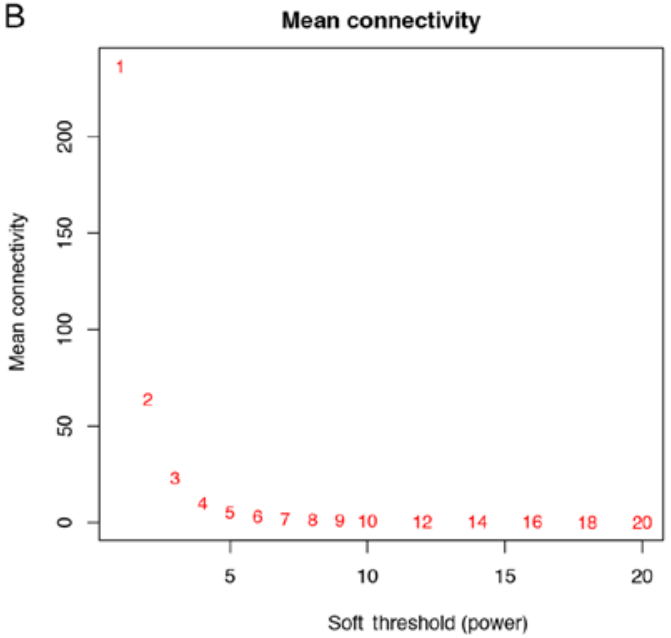

C

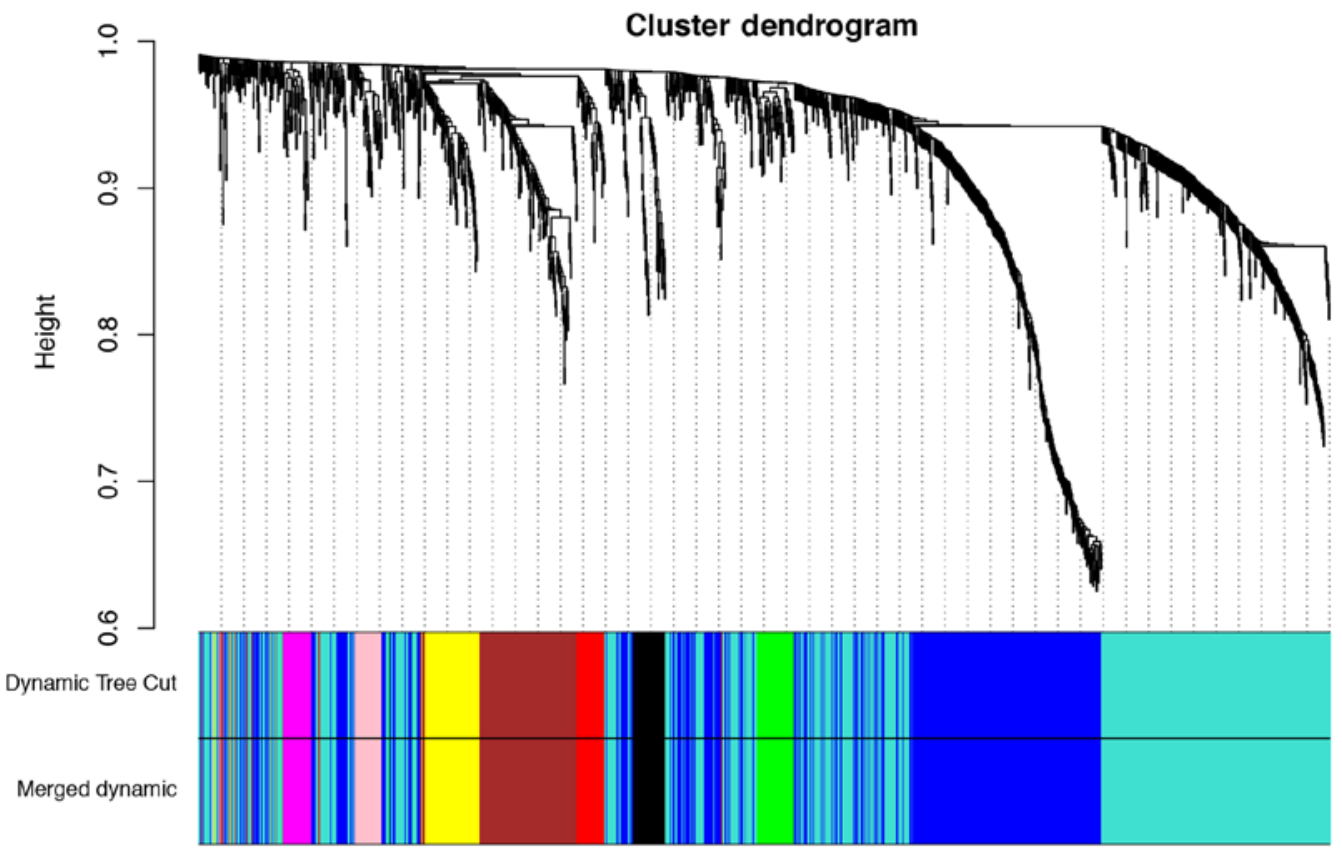

D

\section{Module-trait relationships}

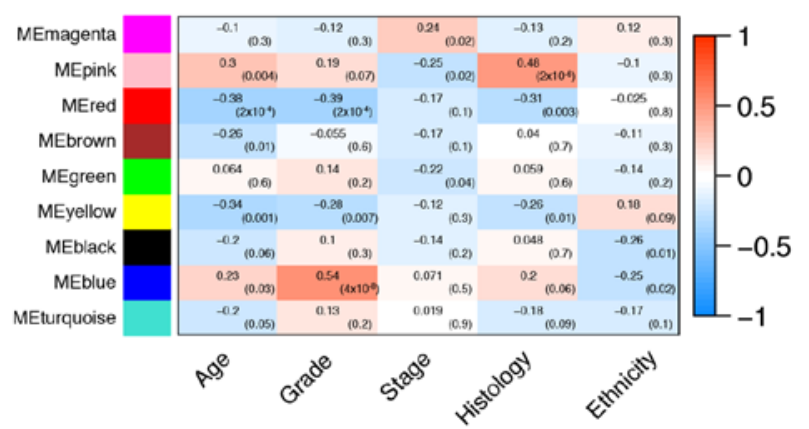

$\mathrm{E} \quad$ Module membership vs. gene significance

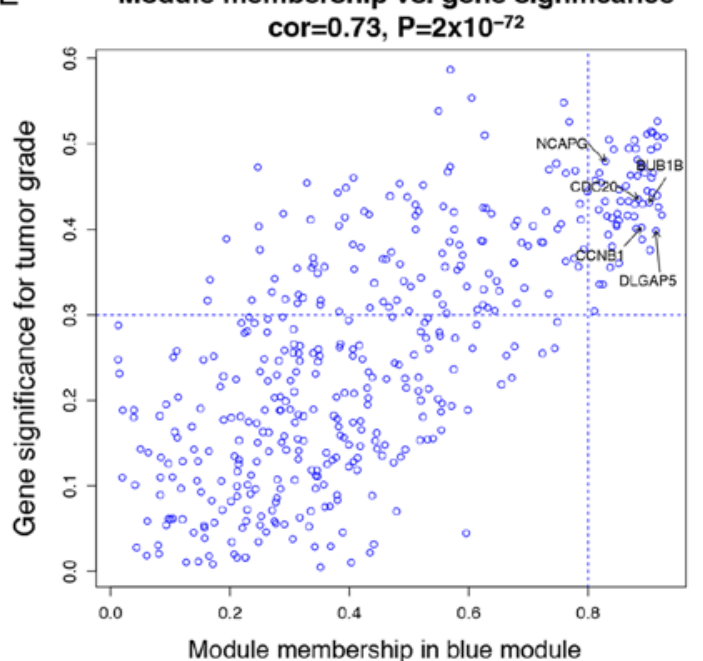

Figure 3. Identification of modules associated with the clinical traits of UCEC by weighted gene co-expression network analysis. (A) Analysis of the scale-free fit index for various soft-thresholding powers ( $\beta$ ). (B) Analysis of the mean connectivity for various soft-thresholding powers. (C) Dendrogram of all differentially expressed genes clustered based on a dissimilarity measure (1-TOM). (D) Heatmap showing the correlation between module eigengenes and clinical traits of UCEC. (E) Scatter plot of module eigengenes in the blue module in the GSE17025 dataset. The hub genes in the module are represented by dots in the upper-right corner of the plot. UCEC, uterine corpus endometrial carcinoma; ME, module eigengene. 
A

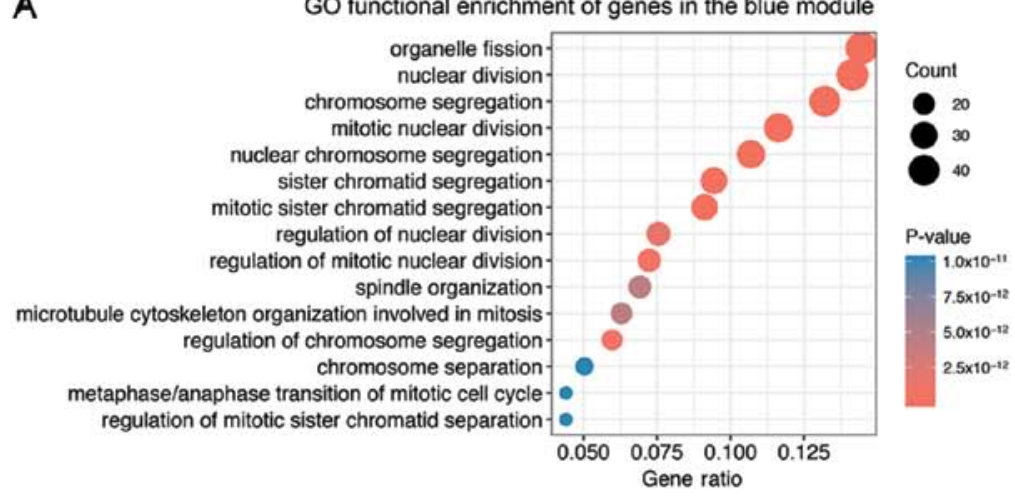

B

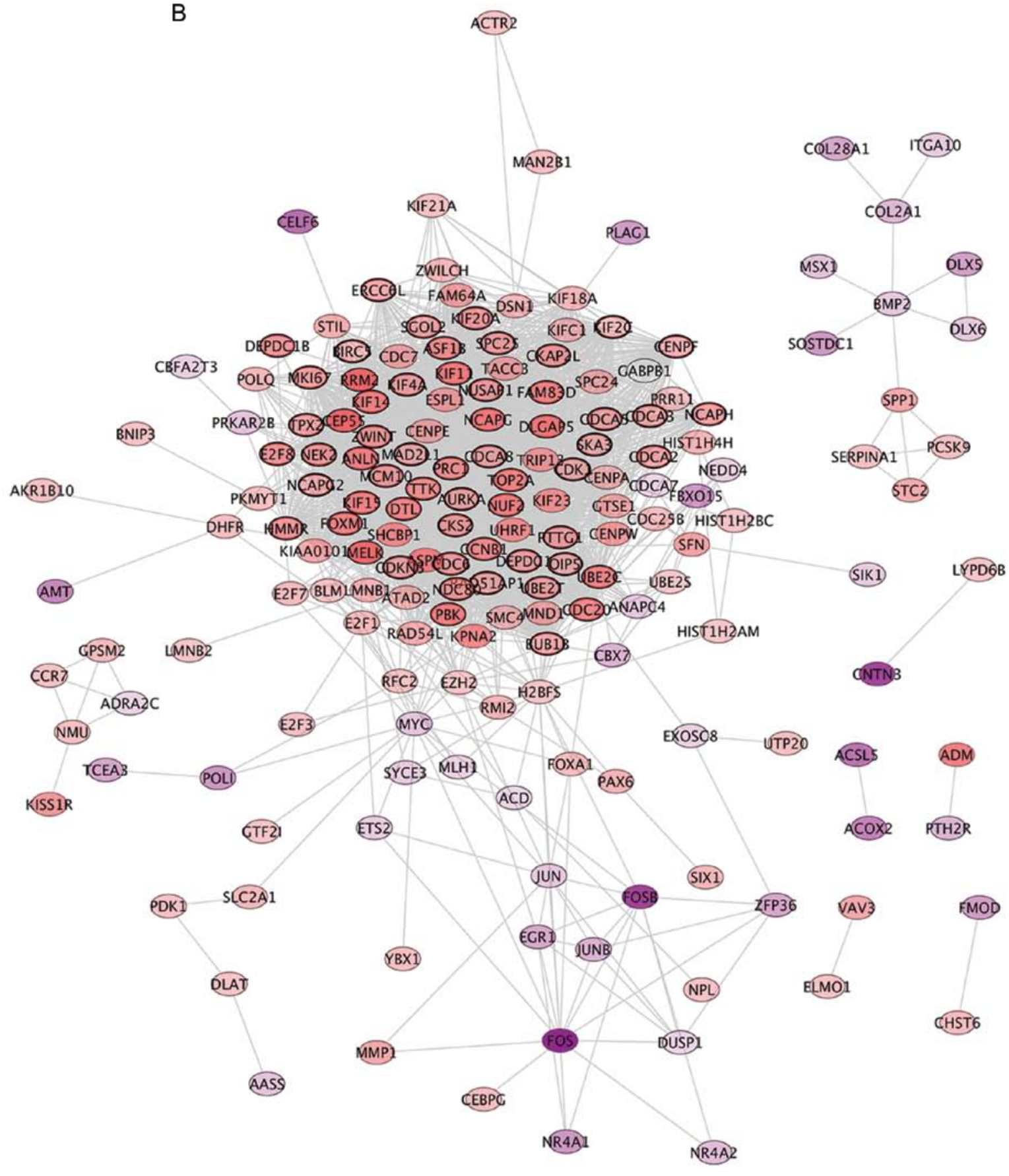

Figure 4. GO functional enrichment analysis and PPI network of genes in the blue module. (A) GO functional enrichment analysis of genes in the blue module. The $\mathrm{x}$-axis shows the gene ratio and the $\mathrm{y}$-axis shows the GO terms. The P-value of each term is colored as shown in the key. The size of dot is proportional to the number of genes. (B) PPI network of genes in the blue module. The color intensity in each node is proportional to the variation of expression (upregulated genes are shown in red and downregulated genes are shown in purple). The nodes with a bold circle represent network hub genes identified by weighted gene co-expression network analysis. PPI, protein-protein interaction; GO, Gene Ontology. 
Table I. Top 25 Reactome pathways of tumor grade-associated genes in uterine corpus endometrial carcinoma.

\begin{tabular}{|c|c|c|c|c|}
\hline Pathway & Description & Count & P-value & $\begin{array}{l}\text { False discovery } \\
\text { rate }\end{array}$ \\
\hline R-HSA-69278 & Cell Cycle, Mitotic & $65 / 570$ & $1.11 \times 10^{-16}$ & $4.36 \times 10^{-14}$ \\
\hline R-HSA-1640170 & Cell Cycle & $74 / 682$ & $1.11 \times 10^{-16}$ & $4.36 \times 10^{-14}$ \\
\hline R-HSA-69620 & Cell Cycle Checkpoint & $36 / 279$ & $2.12 \times 10^{-11}$ & $5.57 \times 10^{-9}$ \\
\hline R-HSA-69618 & Mitotic Spindle Checkpoint & $21 / 110$ & $4.69 \times 10^{-10}$ & $9.20 \times 10^{-8}$ \\
\hline R-HSA-141444 & $\begin{array}{l}\text { Amplification of signal from unattached kinetochores } \\
\text { via a MAD2 inhibitory signal }\end{array}$ & $19 / 94$ & $1.27 \times 10^{-9}$ & $1.66 \times 10^{-7}$ \\
\hline R-HSA-141424 & Amplification of signal from the kinetochores & $19 / 94$ & $1.27 \times 10^{-9}$ & $1.66 \times 10^{-7}$ \\
\hline R-HSA-2500257 & Resolution of Sister Chromatid Cohesion & $22 / 134$ & $2.78 \times 10^{-9}$ & $3.12 \times 10^{-7}$ \\
\hline R-HSA-68877 & Mitotic Prometaphase & $27 / 207$ & $5.77 \times 10^{-9}$ & $5.65 \times 10^{-7}$ \\
\hline R-HSA-68886 & M Phase & $38 / 390$ & $1.28 \times 10^{-8}$ & $1.12 \times 10^{-6}$ \\
\hline R-HSA-68882 & Mitotic Anaphase & $25 / 208$ & $9.87 \times 10^{-8}$ & $7.54 \times 10^{-6}$ \\
\hline R-HSA-2467813 & Separation of Sister Chromatids & $24 / 194$ & $1.06 \times 10^{-7}$ & $7.54 \times 10^{-6}$ \\
\hline R-HSA-2555396 & Mitotic Metaphase and Anaphase & $25 / 211$ & $1.29 \times 10^{-7}$ & $8.36 \times 10^{-6}$ \\
\hline R-HSA-5663220 & RHO GTPases Activate Formins & $20 / 149$ & $3.54 \times 10^{-7}$ & $2.12 \times 10^{-5}$ \\
\hline R-HSA-195258 & RHO GTPase Effectors & $28 / 316$ & $6.44 \times 10^{-6}$ & $3.60 \times 10^{-4}$ \\
\hline R-HSA-1538133 & G0 and Early G1 & $9 / 38$ & $8.45 \times 10^{-6}$ & $4.39 \times 10^{-4}$ \\
\hline R-HSA-539107 & Activation of E2F1 target genes at G1/S & $9 / 43$ & $2.22 \times 10^{-5}$ & 0.001 \\
\hline R-HSA-69205 & G1/S-Specific Transcription & $9 / 43$ & $2.22 \times 10^{-5}$ & 0.001 \\
\hline R-HSA-1362277 & $\begin{array}{l}\text { Transcription of E2F targets under negative control by } \\
\text { DREAM complex }\end{array}$ & $7 / 25$ & $3.00 \times 10^{-5}$ & 0.001 \\
\hline R-HSA-6791312 & TP53 Regulates Transcription of Cell Cycle Genes & $10 / 65$ & $1.01 \times 10^{-4}$ & 0.004 \\
\hline R-HSA-453279 & Mitotic G1-G1/S phases & $17 / 173$ & $1.28 \times 10^{-4}$ & 0.005 \\
\hline R-HSA-983189 & Kinesins & $10 / 68$ & $1.46 \times 10^{-4}$ & 0.005 \\
\hline R-HSA-156711 & Polo-like kinase mediated events & $6 / 23$ & $1.62 \times 10^{-4}$ & 0.006 \\
\hline R-HSA-2514853 & Condensation of Prometaphase Chromosomes & $5 / 15$ & $1.88 \times 10^{-4}$ & 0.006 \\
\hline R-HSA-179409 & APC-Cdc20 mediated degradation of Nek2A & $6 / 25$ & $2.53 \times 10^{-4}$ & 0.008 \\
\hline R-HSA-69481 & G2/M Checkpoints & $15 / 154$ & $3.43 \times 10^{-4}$ & 0.011 \\
\hline
\end{tabular}

$(\mathrm{P}=0.0307), \mathrm{CDC} 20(\mathrm{P}=0.0011)$, DLGAP5 $(\mathrm{P}=0.0036)$ and NCAPG $(\mathrm{P}=0.0056)$ were significantly associated with the OS rate of patients with UCEC, suggesting that patients with UCEC with higher expression of the five genes exhibited a poor prognosis (Fig. 6F-J).

Verification of the expression of hub genes at the protein level. To investigate the protein expression patterns of the five hub genes in samples of patients with UCEC and further interrogate the prognostic significance of these genes, the expression levels of CCNB1, CDC20, DLGAP5 and NCAPG were analyzed in The Human Protein Atlas database (Fig. 7). The expression level of BUB1B protein was not analyzed as its information was not available in The Human Protein Atlas database. In agreement with the earlier analysis, this analysis revealed that the CCNB1, CDC20, DLGAP5 and NCAPG proteins were expressed at higher levels in UCEC tissues compared with those in normal tissues. Analysis of IHC images revealed that CCNB1, CDC20, DLGAP5 and NCAPG proteins were localized in the cytoplasm and cell surface membranes of UCEC cells. Notably, CDC20 exhibited a strong nuclear localization. All patient information obtained from The Human Protein Atlas database is provided in Table III.

\section{Discussion}

UCEC and normal endometrial tissues exhibit a high degree of genetic heterogeneity (6). Although $75 \%$ of UCEC cases are diagnosed at stage I, a significant subset of patients with UCEC are identified at a high-grade disease stage (4). The current therapeutic strategies for this condition do not effectively control progression of UCEC (50). To improve disease outcomes, novel therapeutic strategies with higher specificity and efficacy are urgently needed, particularly for high-grade UCEC. Previous studies have suggested that activated leukocyte cell adhesion molecule and L1 cell adhesion molecule are key players in the oncogenesis of early stage and moderately to poorly differentiated UCEC, respectively $(50,51)$. In the present study, WGCNA revealed five tumor grade-related genes. The association of these genes with UCEC was validated by evaluating their mRNA and protein levels in UCEC samples. The current results demonstrate that this group of tumor grade-related genes plays a critical role in the development of UCEC. These genes can be used to predict prognosis and are ideal therapeutic targets in UCEC.

WGCNA has previously been employed to identify genes associated with clinical features from various publicly 
A

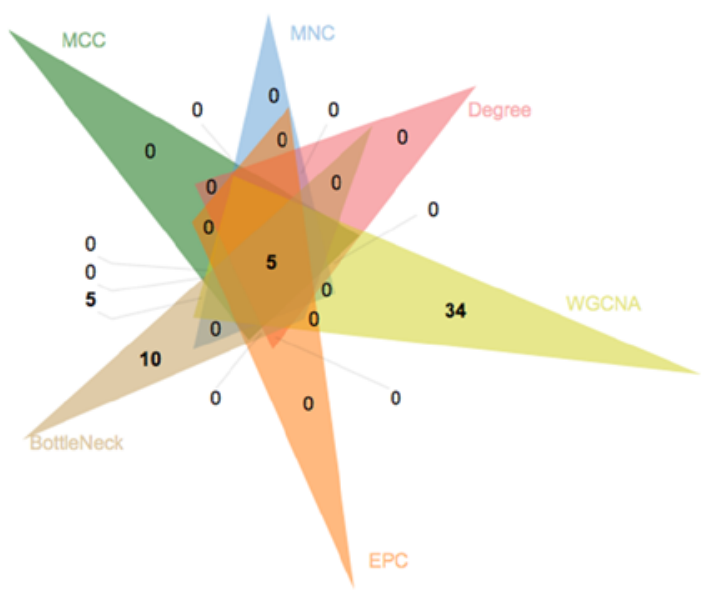

B

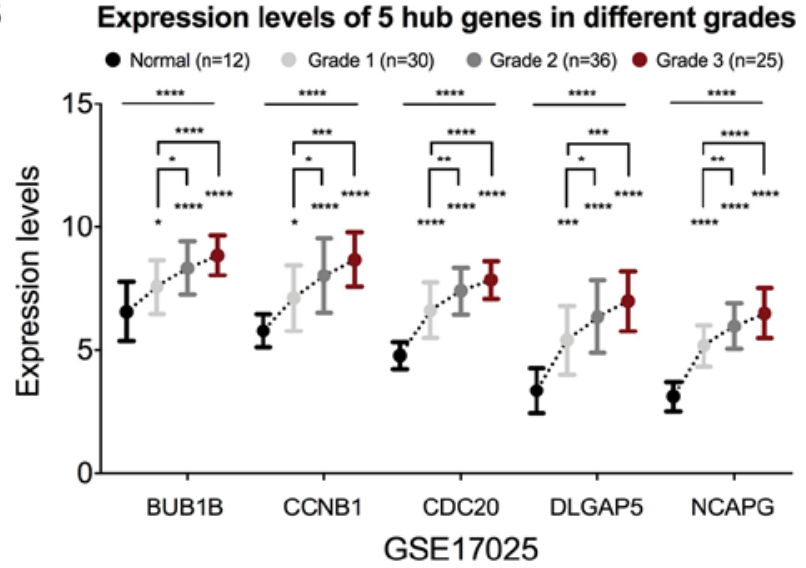

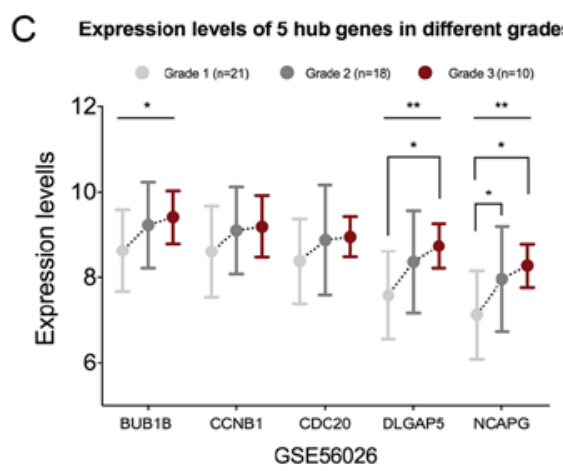

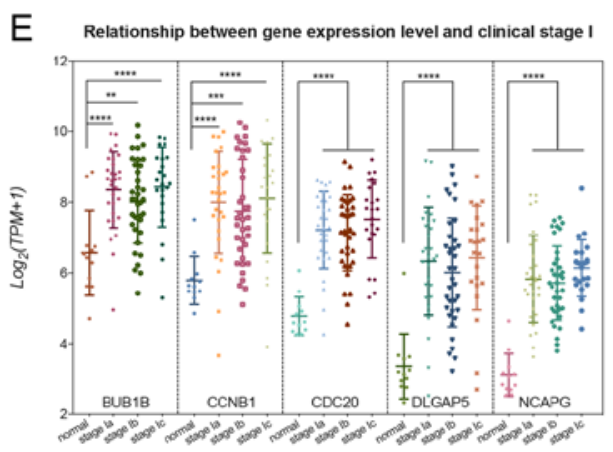

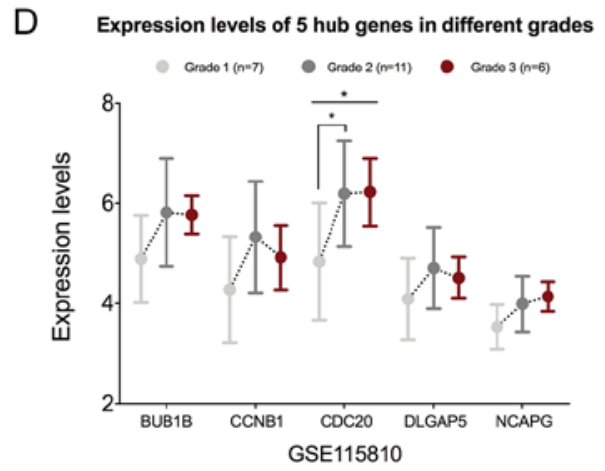

$F$

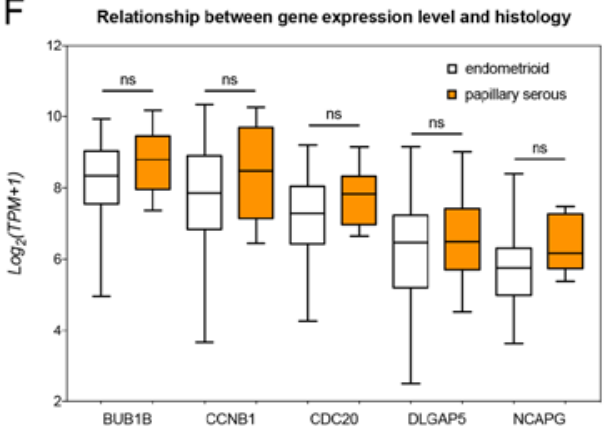

G
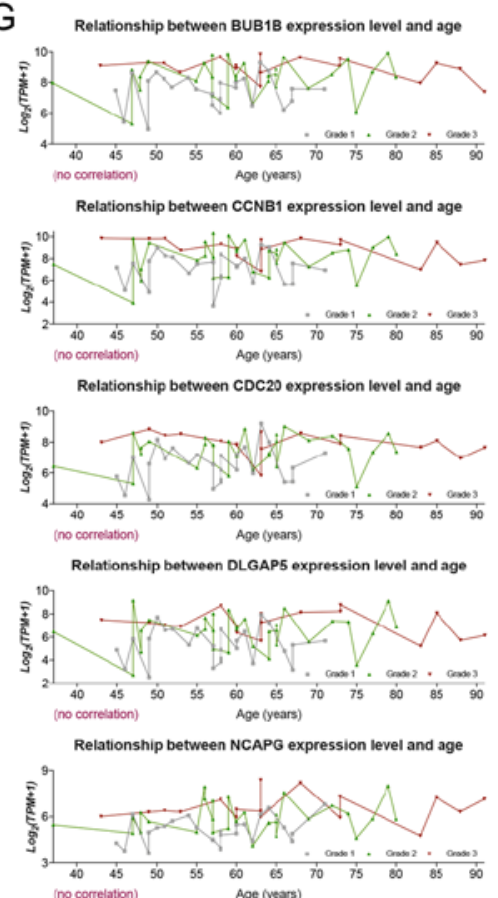

Figure 5. Identification and validation of real hub genes associated with tumor grades. (A) The five common hub genes in both the co-expression network and protein-protein interaction network were regarded as real hub genes. (B) Expression levels of the five hub genes in 103 samples with different grades. (C) Expression levels of the five hub genes in the GSE56026 dataset with different grades. (D) Expression levels of the five hub genes in the GSE115810 dataset with different grades. (E) Five genes were expressed at a significantly higher in stage Ia, Ib and Ic compared with those in normal tissues; however, there was no significant difference in their expression among the stage Ia, Ib and Ic EC samples. (F) There was no significant difference in gene expression levels between endometrioid EC and papillary serous EC. (G) There was no correlation between gene expression levels and age of patients. ${ }^{*} \mathrm{P}<0.05$; ${ }^{* *} \mathrm{P}<0.01 ;{ }^{* * *} \mathrm{P}<0.001$; ${ }^{* * * *} \mathrm{P}<0.0001$. EC, endometrial carcinoma; MCC, maximal clique centrality; MNC, maximum neighborhood component; EPC, edge percolated component; BN, bottleneck; BUB1B, BUB1 mitotic checkpoint serine/threonine kinase B; CCNB1, cyclin B1; CDC20, cell-division cycle protein 20; DLGAP5, DLG-associated protein 5; NCAPG, non-SMC condensing I complex subunit; ns, not significant; WCGNA, weighted gene co-expression network analysis.

available databases. A previous study used this method to identify 5 EC-related modules and prioritize 11 aberrantly expressed genes, and it was determined that only 6 genes had mutations with relevance to EC (25). The present study is more extensive than this previous study. First, the associations between DEGs and tumor clinical features were analyzed. This analysis uncovered the blue module, which was significantly associated with high tumor grades, and 60 hub genes were identified. Furthermore, MCC, MNC, degree, EPC and BN methods of Cytoscapes's CytoHubba plug-in were employed to select high-ranking genes in the blue module with the highest connectivity scores from a PPI network of the 428 DEGs. This analysis produced five overlapping hub genes in the co-expression network and PPI network. These five candidates were found to be strongly associated with higher tumor grades. Survival analysis revealed that high expression of these genes predicted a poor OS rate in UCEC. Further analysis demonstrated that mRNA expression levels of these genes were in line with the results obtained from the gene co-expression analysis. Although the expression level of BUB1B protein was not available in The Human Protein Atlas database, the other four genes were found to be upregulated in UCEC tissues. However, the underlying mechanisms explaining how these genes impact the progression and prognosis of UCEC remain unknown.

BUB1B has been reported to serve an important role in the mitotic checkpoint (52). However, it is currently unknown how 
Table II. Reactome pathways of 5 hub genes in uterine corpus endometrial carcinoma.

\begin{tabular}{|c|c|c|c|c|}
\hline Pathway & Description & Enriched genes & P-value & FDR \\
\hline R-HSA-68877 & Mitotic Prometaphase & $\begin{array}{l}\text { BUB1B, CCNB1, } \\
\text { CDC } 20, \text { NCAPG }\end{array}$ & $1.62 \times 10^{-6}$ & $6.94 \times 10^{-5}$ \\
\hline R-HSA-69278 & Cell Cycle, Mitotic & $\begin{array}{l}\text { BUB1B, CCNB1, } \\
\text { CDC20, NCAPG }\end{array}$ & $2.21 \times 10^{-6}$ & $6.94 \times 10^{-5}$ \\
\hline R-HSA-1640170 & Cell Cycle & $\begin{array}{l}\text { BUB1B, CCNB1, } \\
\text { CDC } 20, \text { NCAPG }\end{array}$ & $5.33 \times 10^{-6}$ & $6.94 \times 10^{-5}$ \\
\hline R-HSA-9013508 & $\begin{array}{l}\text { NOTCH3 Intracellular Domain } \\
\text { Regulates Transcription }\end{array}$ & DLGAP5 & $1.38 \times 10^{-4}$ & $4.14 \times 10^{-4}$ \\
\hline R-HSA-9012852 & Signaling by NOTCH3 & DLGPA5 & $4.20 \times 10^{-4}$ & 0.001 \\
\hline
\end{tabular}

FDR, false discovery rate; BUB1B, BUB1 mitotic checkpoint serine/threonine kinase B; CCNB1, cyclin B1; CDC20, cell-division cycle protein 20; DLGAP5, DLG-associated protein 5; NCAPG, non-SMC condensing I complex subunit.

its aberrant expression impacts tumorigenesis. Overexpression of BUB1B has been demonstrated to be strongly associated with advanced tumor stage, poor OS rate and high tumor recurrence rate in various tumors, including pancreatic ductal adenocarcinoma, gastric cancer, bladder cancer and liver cancer (53-56). An in vitro study on verteporfin-treated type 1 EC cell lines (HEC-1-B cells) demonstrated that BUB1B regulates the progression of the mitotic checkpoint (57). By contrast, lower levels of BUB1B have been associated with a poor OS rate in colon adenocarcinoma (52).

CDC20, in cooperation with other factors, has been reported to be an important modulator of the cell cycle and is associated with advanced tumor stages and higher grades in multiple cancer types, including pancreatic (58), breast (59) and colorectal cancer (60). A previous study suggested that low CDC20 expression is associated with an improved OS rate and that knockdown of CDC20 expression suppresses proliferation of EC cells (61).

The present study identified several genes, including BUB1B and CDC20, that when upregulated, were associated with poor OS in UCEC. Functional enrichment analysis results demonstrated that these genes are mainly involved in the mitotic prometaphase pathway during the $\mathrm{M}$ phase of the cell cycle. Taken together, these observations point to a potential prognostic value for BUB1B and CDC20 in UCEC.

The current study also revealed that CCNB1 could be a potential prognostic biomarker for UCEC. The CCNB1 gene encodes an important regulator of the cell cycle and is associated with cyclin-dependent kinase 1 , and it is also known to regulate the $G_{2} / M$ transition during mitosis (62). It has been previously reported that CCNB1 expression is associated with poorly differentiated EC (7). Integrated bioinformatics analysis methods were used to examine TCGA and GEO datasets (26). This led to the identification of 11 hub genes that are closely associated with EC tumorigenesis, including CCNB1 (26). Several reports have indicated that CCNB1 serves as a tumor antigen in cancer immunotherapy $(62,63)$. In hepatocellular carcinoma (HCC), CCNB1 regulates tumorigenesis and predicts the prognosis of the disease (63). However, the role of CCNB1 in UCEC has not been sufficiently investigated.
NCAPG participates in the condensation and stabilization of chromosomes during cell division (64). To the best of our knowledge, this gene has also not previously been characterized in UCEC. Overexpression of NCAPG has been shown to be correlated with that of CCNB1 (65). NCAPG silencing induces HCC cell entry into mitosis leading to inhibition of cell growth and proliferation both in vitro and in vivo (65). In patients with HCC, high expression of NCAPG is associated with poor OS and high recurrence rates (65). The present functional enrichment analyses revealed that CCNB1 and NCAPG are upregulated in UCEC and are involved in cell cycle-related pathways. Taken together, these data suggest that CCNB1 and NCAPG are promising prognostic and therapeutic targets in UCEC.

In the current analysis, DLGAP5 was identified as a hub gene associated with higher tumor grade in UCEC. This gene has previously been shown to play a crucial role in the $\mathrm{G}_{2} / \mathrm{M}$ transition in the cell cycle (66). The role of DLGAP5 in UCEC has previously been investigated. Using bioinformatics, DLGAP5 was reported to be among the $11 \mathrm{EC}$ tumorigenic and prognostic genes (25). In this previous study, it was reported that DLGAP5 expression in EC tissues predicts a poor OS rate, which agrees with the present findings. In addition, aberrant expression of DLGAP5 was found to be correlated with higher tumor grades in UCEC as revealed by co-expression network and PPI network analysis (25). The present study also identified that DLGAP5 is associated with the NOTCH3 intracellular domain, which regulates gene transcription (49). While, to the best of our knowledge, only a few studies have investigated the molecular mechanisms of DLGAP5 in EC, DLGAP5 has been studied extensively in other cancer types. It has been reported that, as a NOTCH3 target gene, DLGAP5 silencing inhibits tumorigenicity and proliferation of cancer cells by arresting the cell cycle in the $\mathrm{G}_{2} / \mathrm{M}$ phase in ovarian cancer (49). Another study suggested that DLGAP5 is associated with aggressive colorectal cancer, and its overexpression predicted a poor OS rate in the lower crypt-like subtype of colorectal cancer (66). In summary, the present findings and previous studies suggest that DLGAP5 may be a potential prognostic biomarker of UCEC.

The present study has several innovative aspects. To the best of our knowledge, this is the first report that has 

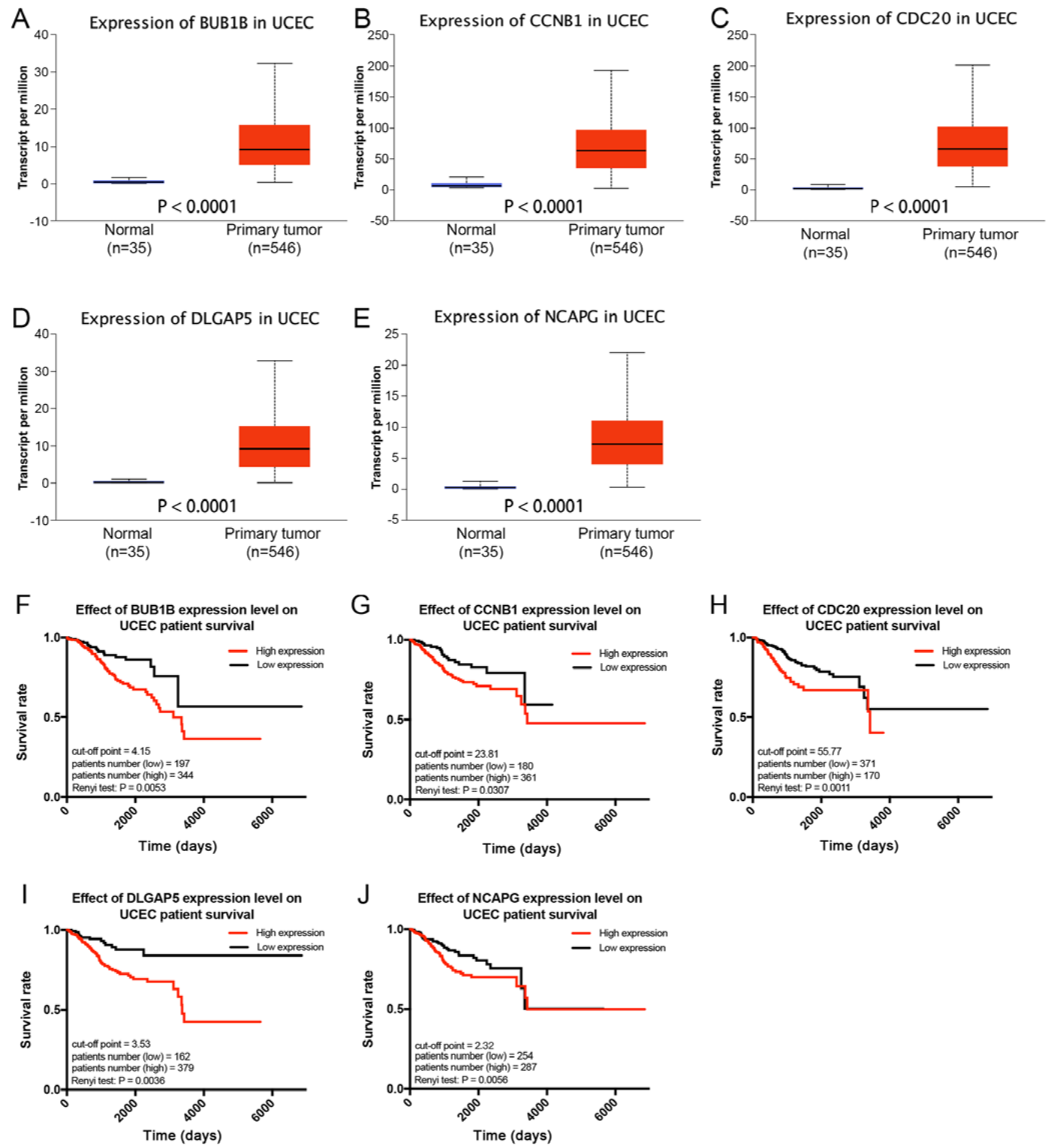

Figure 6. Validation and survival analysis of real hub genes in UCEC based on TCGA database. The mRNA expression levels of (A) BUB1B, (B) CCNB1, (C) CDC20, (D) DLGAP5 and (E) NCAPG were significantly higher in UCEC tissues compared with those in normal tissues. Kaplan-Meier plots showing the association of (F) BUB1B, (G) CCNB1, (H) CDC20, (I) DLGAP5 and (J) NCAPG expression levels with the survival of 541 patients with UCEC, with the best expression cut-off point according to the clinical data on TCGA. BUB1B, BUB1 mitotic checkpoint serine/threonine kinase B; CCNB1, cyclin B1; CDC20, cell-division cycle protein 20; DLGAP5, DLG-associated protein 5; NCAPG, non-SMC condensing I complex subunit; UCEC, uterine corpus endometrial carcinoma; TCGA, The Cancer Genome Atlas.

investigated the associations between DEGs and clinical features of UCEC cases. Furthermore, three gene expression microarray datasets from the GEO database and one RNA-seq dataset from TCGA database were combined to comprehensively identify DEGs that are relevant to the progression and prognosis of UCEC. Indeed, the selection of samples was crucial to the present analysis. A small sample size and the absence of the normal group could have an effect on obtaining reliable DEGs. The GSE17025 dataset consisted of 91 EC samples and 12 normal endometrium samples. However, the GSE56026 dataset did not contain normal endometrium samples, and the GSE115810 
Table III. Immunohistochemistry staining characteristics of the hub genes from The Human Protein Atlas database.

A, Normal tissue

\begin{tabular}{lclcllll}
\hline Gene & Patient ID & Sex & Age, years & Staining & Intensity & Quantity & Location \\
\hline CCNB1 & 2361 & Female & 33 & Medium & Strong & Rare & Cytoplasmic membranous \\
CDC20 & 2941 & Female & 33 & Medium & Strong & $<25 \%$ & Nuclear \\
DLGAP5 & 1978 & Female & 72 & Low & Moderate & $<25 \%$ & Cytoplasmic membranous \\
NCAPG & 3313 & Female & 39 & Medium & Moderate & $>75 \%$ & Cytoplasmic membranous \\
\hline
\end{tabular}

$\mathrm{B}$, Uterine corpus endometrial carcinoma tissue

\begin{tabular}{lccclccc}
\hline Gene & Patient ID & Sex & Age, years & Staining & Intensity & Quantity & Location \\
\hline CCNB1 & 167 & Female & 75 & Medium & Moderate & $>75 \%$ & Cytoplasmic membranous \\
CDC20 & 1165 & Female & 75 & High & Strong & $75-25 \%$ & Cytoplasmic membranous \\
nuclear & & & & & & \\
DLGAP5 & 1750 & Female & 83 & Medium & Strong & $<25 \%$ & Cytoplasmic membranous \\
NCAPG & 3036 & Female & 32 & High & Strong & $>75 \%$ & Cytoplasmic membranous
\end{tabular}

CCNB1, cyclin B1; CDC20, cell-division cycle protein 20; DLGAP5, DLG-associated protein 5; NCAPG, non-SMC condensing I complex subunit.

A

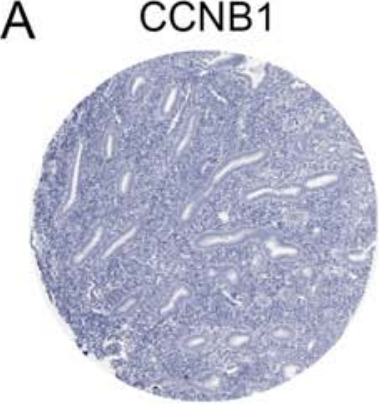

Normal tissue

Human Protein Atlas: (https://www.proteinatlas.org// tissue/endometrium\#img)

C

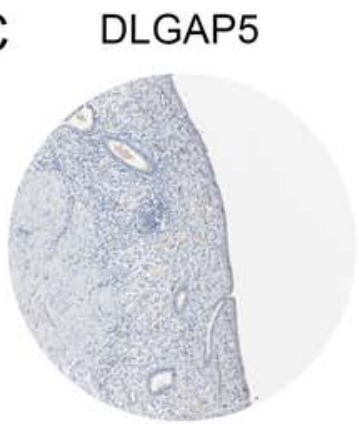

Normal tissue

Human Protein Atlas: (https://www.proteinatlas.org/ tissue/endometrium\#img)
CCNB1

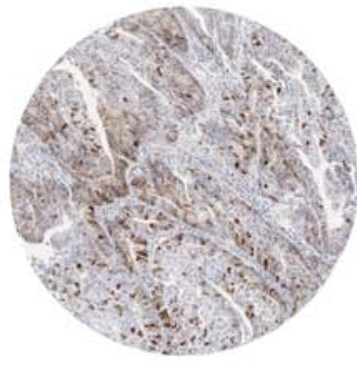

EC tissue

Human Protein Atlas: (https://www proteinatlas.org/ pathology/endometial+cancer\#img)

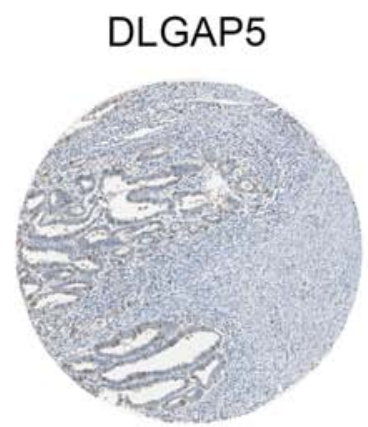

EC tissue

Human Protein Atlas: (https://www.proteinatlas.org/ ENSG00000126787-DLGAP5/

pathology/endometial+cancer\#img)
B $\quad$ CDC20

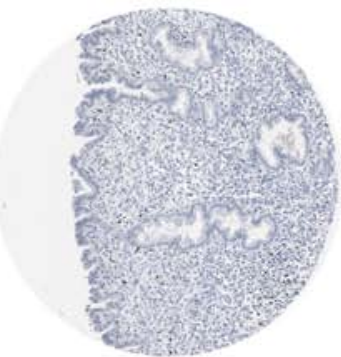

Normal tissue

Human Protein Atlas: (https://www.proteinatlas.org/ tissue/endometrium\#img)

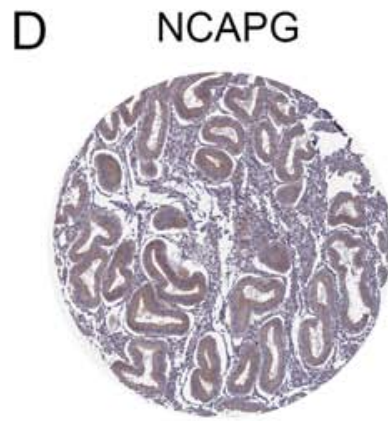

Normal tissue

Human Protein Atlas: (https://www.proteinatlas.org/ tissue/endometrium\#img)
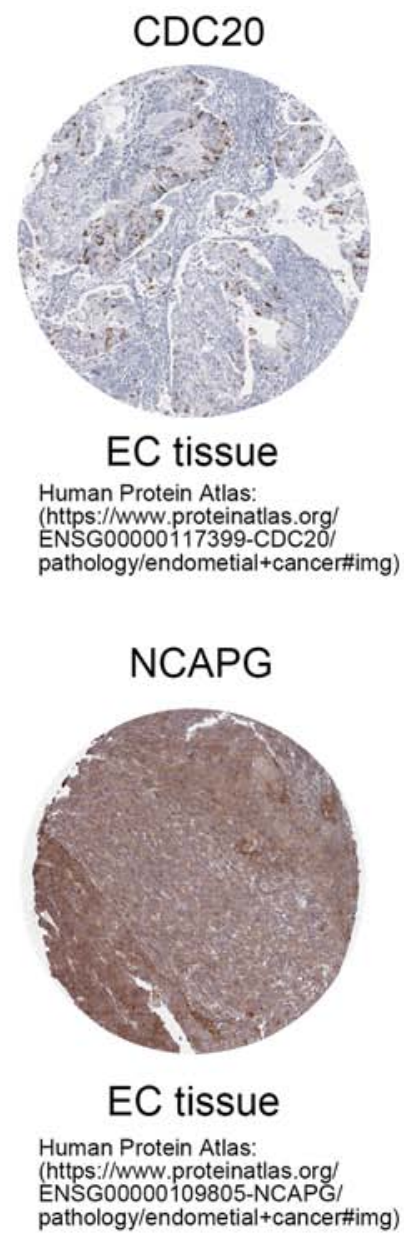

Figure 7. Validation of protein expression levels of CCNB1, CDC20, DLGAP5 and NCAPG. The (A) CCNB1, (B) CDC20, (C) DLGAP5 and (D) NCAPG proteins were expressed at higher levels in EC tissues compared with those in normal tissues, as revealed by The Human Protein Atlas database (http://www. proteinatlas.org). CCNB1, cyclin B1; CDC20, cell-division cycle protein 20; DLGAP5, DLG-associated protein 5; NCAPG, non-SMC condensing I complex subunit; EC, endometrial carcinoma. 
dataset contained only three normal samples. WGCNA requires a sample size of at least 15 for each group. Among the 91 EC samples in the GSE17025 dataset, there were 30 grade I samples, 36 grade II samples and 25 grade III samples. In addition, TCGA UCEC data contained more complete clinical information on the patients with UCEC, such as survival time and survival status, which were of great importance for the OS analysis in the study. In addition, survival data of patients with UCEC were missing from the GSE17025 dataset. Therefore, after comprehensive consideration, it was considered that the GSE17025 dataset was more suitable to be analyzed as a training dataset, whereas the other two gene expression microarray datasets were more suitable as testing datasets, and TCGA data was used for the OS analysis to evaluate the hub genes. Furthermore, two functional enrichment analysis databases and five methods in Cytoscape's CytoHubba plug-in were co-utilized to rigorously screen for tumor grade-related hub genes. A limitation of the present approach is that the precise molecular mechanism by which these tumor grade-related genes impact the progression and prognosis of UCEC could not be determined.

In conclusion, WGCNA revealed five tumor grade-related genes from 1,447 DEGs between UCEC tissues and normal endometrial tissues. The tumorigenic role of these hub genes was validated at the RNA and protein levels. High expression of the five hub genes was associated with poor OS rate. Furthermore, these genes demonstrated potential to predict the prognosis of UCEC and may be therapeutic targets in this type of cancer. Further experimental studies are required to experimentally validate these findings.

\section{Acknowledgements}

The results published in the present study are in whole or part based upon data generated by The Cancer Genome Atlas Research Network (https://www.cancer.gov/tcga), the Gene Expression Omnibus database (http://www.ncbi.nlm.nih.gov/ geo/) and The Human Protein Atlas database (http://www. proteinatlas.org).

\section{Funding}

No funding was received.

\section{Availability of data and materials}

The datasets generated and/or analyzed during the present study are available in the Gene Expression Omnibus database (GSE17025, GSE56026 and GSE115810; http://www.ncbi.nlm. nih.gov/geo/), The Cancer Genome Atlas database (UCEC RNA-sequencing; https://www.cancer.gov/tcga) and The Human Protein Atlas database (http://www.proteinatlas.org).

\section{Authors' contributions}

JB and YX designed the study. YX and FW downloaded all datasets used in the current study and analyzed the differentially expressed genes between UCEC and normal endometrial tissues. QP and YL participated in the interpretation of the data, prepared the figures and revised the manuscript. JB and YX drafted the manuscript and revised the manuscript. All authors contributed to manuscript writing. All authors read and approved the final manuscript.

\section{Ethics approval and consent to participate}

Not applicable.

\section{Patient consent for publication}

Not applicable.

\section{Competing interests}

The authors declare that they have no competing interests.

\section{References}

1. Siegel RL, Miller KD and Jemal A: Cancer statistics, 2018. CA Cancer J Clin 68: 7-30, 2018.

2. Pecorelli S: Revised FIGO staging for carcinoma of the vulva, cervix, and endometrium. Int J Gynaecol Obstet 105: 103-104, 2009.

3. Murali R, Soslow RA and Weigelt B: Classification of endometrial carcinoma: More than two types. Lancet Oncol 15: e268-e278, 2014.

4. Creasman WT, Odicino F, Maisonneuve P, Quinn MA, Beller U, Benedet JL, Heintz AP, Ngan HY and Pecorelli S: Carcinoma of the corpus uteri. FIGO 26th Annual report on the results of treatment in gynecological cancer. Int J Gynaecol Obstet 95 (Suppl 1): S105-S143, 2006.

5. Colombo N, Creutzberg C, Amant F, Bosse T, González-Martín A Ledermann J, Marth C, Nout R, Querleu D, Mirza MR, et al: ESMO-ESGO-ESTRO consensus conference on endometrial cancer: Diagnosis, treatment and follow-up. Radiother Oncol 117: 559-581, 2015.

6. Morice P, Leary A, Creutzberg C, Abu-Rustum N and Darai E: Endometrial cancer. Lancet 387: 1094-1108, 2016.

7. Zang Y, Dong M, Zhang K, Tian W, Wang Y and Xue F: Bioinformatics analysis of key differentially expressed genes in well and poorly differentiated endometrial carcinoma. Mol Med Rep 18: 467-476, 2018.

8. Kaloglu S, Guraslan H, Tekirdag AI, Dagdeviren H and Kaya C: Relation of preoperative thrombocytosis between tumor stage and grade in patients with endometrial cancer. Eurasian J Med 46: 164-168, 2014.

9. Kong A, Johnson N, Kitchener HC and Lawrie TA: Adjuvant radiotherapy for stage I endometrial cancer: An updated Cochrane systematic review and meta-analysis. J Natl Cancer Inst 104: 1625-1634, 2012.

10. Susumu N, Sagae S, Udagawa Y,Niwa K, Kuramoto H, Satoh S and Kudo R; Japanese Gynecologic Oncology Group: Randomized phase III trial of pelvic radiotherapy versus cisplatin-based combined chemotherapy in patients with intermediate- and high-risk endometrial cancer: A Japanese Gynecologic Oncology Group study. Gynecol Oncol 108: 226-233, 2008.

11. Bradford LS, Rauh-Hain JA, Schorge J, Birrer MJ and Dizon DS: Advances in the management of recurrent endometrial cancer. Am J Clin Oncol 38: 206-212, 2015.

12. Tsoref D, Welch S, Lau S, Biagi J, Tonkin K, Martin LA, Ellard S, Ghatage P, Elit L, Mackay HJ, et al: Phase II study of oral ridaforolimus in women with recurrent or metastatic endometrial cancer. Gynecol Oncol 135: 184-189, 2014.

13. Oza AM, Pignata S, Poveda A, McCormack M, Clamp A, Schwartz B, Cheng J, Li X, Campbell K, Dodion P and Haluska FG: Randomized phase II trial of ridaforolimus in advanced endometrial carcinoma. J Clin Oncol 33: 3576-3582, 2015.

14. Matulonis U, Vergote I, Backes F, Martin LP, McMeekin S, Birrer M, Campana F, Xu Y, Egile C and Ghamande S: Phase II study of the PI3K inhibitor pilaralisib (SAR245408; XL147) in patients with advanced or recurrent endometrial carcinoma. Gynecol Oncol 136: 246-253, 2015. 
15. Powell MA, Sill MW, Goodfellow PJ, Benbrook DM,Lankes HA Leslie KK, Jeske Y, Mannel RS, Spillman MA, Lee PS, et al: A phase II trial of brivanib in recurrent or persistent endometrial cancer: An NRG Oncology/Gynecologic Oncology Group Study. Gynecol Oncol 135: 38-43, 2014.

16. Lee PS and Secord AA: Targeting molecular pathways in endometrial cancer: A focus on the FGFR pathway. Cancer Treat Rev 40: 507-512, 2014.

17. Forster MD, Dedes KJ, Sandhu S, Frentzas S, Kristeleit R, Ashworth A, Poole CJ, Weigelt B, Kaye SB and Molife LR: Treatment with olaparib in a patient with PTEN-deficient endometrioid endometrial cancer. Nat Rev Clin Oncol 8: 302-306, 2011.

18. Howitt BE, Shukla SA, Sholl LM, Ritterhouse LL, Watkins JC, Rodig S, Stover E, Strickland KC, D'Andrea AD, Wu CJ, et al: Association of polymerase e-Mutated and microsatellite-instable endometrial cancers with neoantigen load, number of tumor-infiltrating lymphocytes, and expression of PD-1 and PD-L1. JAMA Oncol 1: 1319-1323, 2015.

19. Duran-Sanchon S, Moreno L, Augé JM, Serra-Burriel M, Cuatrecasas M, Moreira L, Martín A, Serradesanferm A, Pozo À, Costa R, et al: Identification and validation of microRNA profiles in fecal samples for detection of colorectal cancer. Gastroenterology 158: 947-957.e4, 2020.

20. Polano M, Chierici M, Dal Bo M, Gentilini D, Cintio FD, Baboci L, Gibbs, DL, Furlanello C and Toffoli G: A Pan-cancer approach to predict responsiveness to immune checkpoint inhibitors by machine learning. Cancers (Basel) 11: 1562, 2019.

21. Zhang K, Li H, Yan Y, Zang Y, Li K, Wang Y and Xue F: Identification of key genes and pathways between type I and type II endometrial cancer using bioinformatics analysis. Oncol Lett 18: 2464-2476, 2019.

22. Chou WC, Cheng AL, Brotto $M$ and Chuang CY: Visual gene-network analysis reveals the cancer gene co-expression in human endometrial cancer. BMC Genomics 15: 300, 2014.

23. Huo X, Sun H, Liu Q, Ma X, Peng P, Yu M, Zhang Y, Cao D and Shen K: Clinical and expression significance of AKT1 by Co-expression network analysis in endometrial cancer. Front Oncol 9: 1147, 2019.

24. Liu Y, Nan F, Lu K, Wang Y, Liu Y, Wei S, Wu R and Wang Y: Identification of key genes in endometrioid endometrial adenocarcinoma via TCGA database. Cancer Biomark 21: 11-21, 2017.

25. Liu J, Zhou S, Li S, Jiang Y, Wan Y, Ma X and Cheng W: Eleven genes associated with progression and prognosis of endometrial cancer (EC) identified by comprehensive bioinformatics analysis. Cancer Cell Int 19: 136, 2019.

26. Liu L, Lin J and $\mathrm{He} \mathrm{H}$ : Identification of potential crucial genes associated with the pathogenesis and prognosis of endometrial cancer. Front Genet 10: 373, 2019.

27. Langfelder $P$ and Horvath S: WGCNA: An R package for weighted correlation network analysis. BMC Bioinformatics 9 : 559, 2008.

28. Chen L, Yuan L, Qian K, Qian G, Zhu Y, Wu CL, Dan HC, Xiao $\mathrm{Y}$ and Wang X: Identification of biomarkers associated with pathological stage and prognosis of clear cell renal cell carcinoma by Co-expression network analysis. Front Physiol 9: $399,2018$.

29. Guo X, Xiao H, Guo S, Dong L and Chen J: Identification of breast cancer mechanism based on weighted gene coexpression network analysis. Cancer Gene Ther 24: 333-341, 2017.

30. Zhou XG, Huang XL, Liang SY, Tang SM, Wu SK, Huang TT, Mo ZN and Wang QY: Identifying miRNA and gene modules of colon cancer associated with pathological stage by weighted gene Co-expression network analysis. Onco Targets Ther 11: 2815-2830, 2018

31. Day RS, McDade KK, Chandran UR, Lisovich A, Conrads TP Hood BL, Kolli VS, Kirchner D, Litzi T and Maxwell GL: Identifier mapping performance for integrating transcriptomics and proteomics experimental results. BMC Bioinformatics 12 213,2011

32. Kharma B, Baba T, Matsumura N, Kang HS, Hamanishi J, Murakami R, McConechy MM, Leung S, Yamaguchi K, Hosoe Y, et al: STAT1 drives tumor progression in serous papillary endometrial cancer. Cancer Res 74: 6519-6530, 2014

33. Hermyt E, Zmarzly N, Grabarek B, Kruszniewska-Rajs C, Gola J, Jęda-Golonka A, Szczepanek K, Mazurek U and Witek A: Interplay between miRNAs and Genes Associated with Cell Proliferation in Endometrial Cancer. Int J Mol Sci 20: $6011,2019$.
34. Gautier L, Cope L, Bolstad BM and Irizarry RA: Affy-analysis of Affymetrix GeneChip data at the probe level. Bioinformatics 20 : 307-315, 2004.

35. Stafford P: Methods in microarray normalization. CRC Press, Boca Raton, 2008

36. Irizarry RA, Hobbs B, Collin F, Beazer-Barclay YD, Antonellis KJ, Scherf U and Speed TP: Exploration, normalization, and summaries of high density oligonucleotide array probe level data. Biostatistics 4: 249-264, 2003.

37. Ritchie ME, Phipson B, Wu D, Hu Y, Law CW, Shi W and Smyth GK: Limma powers differential expression analyses for RNA-sequencing and microarray studies. Nucleic Acids Res 43: e47, 2015.

38. Zhang B and Horvath S: A general framework for weighted gene Co-expression network analysis. Stat Appl Genet Mol Biol 4: Article 17, 2005

39. Yip AM and Horvath S: Gene network interconnectedness and the generalized topological overlap measure. BMC Bioinformatics 8: 22, 2007.

40. Ravasz E, Somera AL, Mongru DA, Oltvai ZN and Barabási AL: Hierarchical organization of modularity in metabolic networks. Science 297: 1551-1555, 2002.

41. Yu G, Wang LG, Han Y and He QY: clusterProfiler: An R package for comparing biological themes among gene clusters. OMICS 16: 284-287, 2012.

42. Fabregat A, Sidiropoulos K, Viteri G, Forner O, Marin-Garcia P, Arnau V, D'Eustachio P, Stein L and Hermjakob H: Reactome pathway analysis: A high-performance in-memory approach. BMC Bioinformatics 18: 142, 2017.

43. Jupe S, Ray K, Roca CD, Varusai T, Shamovsky V, Stein L, D'Eustachio P and Hermjakob H: Interleukins and their signaling pathways in the Reactome biological pathway database. J Allergy Clin Immunol 141: 1411-1416, 2018.

44. Szklarczyk D, Gable AL,LyonD, Junge A, WyderS,Huerta-Cepas J, Simonovic M, Doncheva NT, Morris JH, Bork P, et al: STRING v11: Protein-protein association networks with increased coverage, supporting functional discovery in genome-wide experimental datasets. Nucleic Acids Res 47: D607-D613, 2019.

45. Killcoyne S, Carter GW, Smith J and Boyle J: Cytoscape: A community -based framework for network modeling. Methods Mol Biol 563: 219-239, 2009.

46. Chin $\mathrm{CH}$, Chen SH, Wu HH, Ho CW, Ko MT and Lin CY: CytoHubba: Identifying hub objects and sub-networks from complex interactome. BMC Syst Biol 8 (Suppl 4): S11, 2014

47. Uhlén M, Fagerberg L, Hallström BM, Lindskog C, Oksvold P, Mardinoglu A, Sivertsson Å, Kampf C, Sjöstedt E, Asplund A, et al: Proteomics. Tissue-based map of the human proteome. Science 347: 1260419, 2015.

48. Li C: Doubly robust weighted log-rank tests and Renyi-type tests under non-random treatment assignment and dependent censoring. Stat Methods Med Res 28: 2649-2664, 2019.

49. Chen X, Thiaville MM, Chen L, Stoeck A, Xuan J, Gao M, Shih IeM and Wang TL: Defining NOTCH3 target genes in ovarian cancer. Cancer Res 72: 2294-2303, 2012.

50. Devis L, Moiola CP, Masia N, Martinez-Garcia E, Santacana M, Stirbat TV, Brochard-Wyart F, García Á, Alameda F, Cabrera S, et al: Activated leukocyte cell adhesion molecule (ALCAM) is a marker of recurrence and promotes cell migration, invasion, and metastasis in early-stage endometrioid endometrial cancer. J Pathol 241: 475-487, 2017.

51. Klat J, Mladenka A, Dvorackova J, Bajsova S and Simetka O: L1CAM as a negative prognostic factor in endometrioid endometrial adenocarcinoma FIGO Stage IA-IB. Anticancer Res 39: 421-424, 2019.

52. Shichiri M, Yoshinaga K, Hisatomi H, Sugihara K and Hirata Y: Genetic and epigenetic inactivation of mitotic checkpoint genes hBUB1 and hBUBR1 and their relationship to survival. Cancer Res 62: 13-17, 2002

53. Dong S, Huang F, Zhang $\mathrm{H}$ and Chen Q: Overexpression of BUB1B, CCNA2, CDC20, and CDK1 in tumor tissues predicts poor survival in pancreatic ductal adenocarcinoma. Biosci Rep 39: BSR20182306, 2019.

54. Ando K, Kakeji Y, Kitao H, Iimori M,Zhao Y, Yoshida R, Oki E, Yoshinaga K, Matumoto T, Morita M, et al: High expression of BUBR1 is one of the factors for inducing DNA aneuploidy and progression in gastric cancer. Cancer Sci 101: 639-645, 2010.

55. Yamamoto Y, Matsuyama H, Chochi Y, Okuda M, Kawauchi S, Inoue R, Furuya T, Oga A, Naito K and Sasaki K: Overexpression of BUBR1 is associated with chromosomal instability in bladder cancer. Cancer Genet Cytogenet 174: 42-47, 2007. 
56. Liu AW, Cai J, Zhao XL, Xu AM, Fu HQ, Nian H and Zhang SH: The clinicopathological significance of BUBR1 overexpression in hepatocellular carcinoma. J Clin Pathol 62: 1003-1008, 2009.

57. Bang LG, Dasari VR, Kim D and Gogoi RP: Differential gene expression induced by Verteporfin in endometrial cancer cells. Sci Rep 9: 3839, 2019.

58. Chang DZ, Ma Y, Ji B, Liu Y, Hwu P, Abbruzzese JL, Logsdon C and Wang H: Increased CDC20 expression is associated with pancreatic ductal adenocarcinoma differentiation and progression. J Hematol Oncol 5: 15, 2012.

59. Yuan B, Xu Y, Woo JH, Wang Y, Bae YK, Yoon DS, Wersto RP, Tully E, Wilsbach K and Gabrielson E: Increased expression of mitotic checkpoint genes in breast cancer cells with chromosomal instability. Clin Cancer Res 12: 405-410, 2006.

60. Wu WJ, Hu KS, Wang DS, Zeng ZL, Zhang DS, Chen DL, Bai L and Xu RH: CDC20 overexpression predicts a poor prognosis for patients with colorectal cancer. J Transl Med 11: 142, 2013.

61. Huo X, Sun H, Cao D, Yang J,Peng P, Yu Mand Shen K: Identification of prognosis markers for endometrial cancer by integrated analysis of DNA methylation and RNA-Seq data. Sci Rep 9: 9924, 2019.

62. Egloff AM, Vella LA and Finn OJ: Cyclin B1 and other cyclins as tumor antigens in immunosurveillance and immunotherapy of cancer. Cancer Res 66: 6-9, 2006.
63. Gu J, Liu X, Li J and He Y: MicroRNA-144 inhibits cell proliferation, migration and invasion in human hepatocellular carcinoma by targeting CCNB1. Cancer Cell Int 19: 15, 2019.

64. Eberlein A, Takasuga A, Setoguchi K, Pfuhl R, Flisikowski K, Fries R, Klopp N, Fürbass R, Weikard R and Kühn C: Dissection of genetic factors modulating fetal growth in cattle indicates a substantial role of the non-SMC condensin I complex, subunit G (NCAPG) gene. Genetics 183: 951-964, 2009.

65. Zhang Q, Su R, Shan C, Gao C and Wu P: Non-SMC condensin I Complex, Subunit G (NCAPG) is a novel mitotic gene required for hepatocellular cancer cell proliferation and migration. Oncol Res 26: 269-276, 2018.

66. Branchi V, Garcia SA, Radhakrishnan P, Győrffy B, Hissa B, Schneider M, Reißfelder C and Schölch S: Prognostic value of DLGAP5 in colorectal cancer. Int J Colorectal Dis 34: 1455-1465, 2019.

(i) $(9)$ This work is licensed under a Creative Commons Attribution-NonCommercial-NoDerivatives 4.0 International (CC BY-NC-ND 4.0) License. 